\title{
L'impact des budgets sur les soins ambulatoires
}

\author{
Nora Willea, Jürg Schlup \\ ${ }^{a}$ Dr phil., collaboratrice scientifique du président; ${ }^{b}$ Dr méd., président de la FMH
}

Actuellement en Suisse, toutes sortes de "freins aux dépenses de santé» ou de façon explicite des «budgets globaux» sont régulièrement recommandés pour les soins ambulatoires. Mais l'année dernière, l’impact négatif de ces budgets sur la pratique quotidienne des médecins a pourtant été relayé dans plusieurs médias allemands.

A l'automne 2017, le Dr Günter Krause, généraliste et médecin de campagne, a défrayé la chronique en Allemagne lorsqu'il a annoncé vouloir brûler publiquement son attestation de médecin conventionné - c'està-dire l'équivalent allemand de son autorisation de facturer à la charge de l'assurance-maladie obligatoire. Suite à ses études de médecine et sa spécialisation, ce médecin de 38 ans, décrit comme un "personnage agréable à l'humour décapant» [1], est retourné dans son village natal, Hainewalde, avec ses 1500 habitants, où il a repris le cabinet de sa mère en 2014 après quelques années de collaboration familiale. En octobre 2017, son existence professionnelle ne lui semblait plus valoir la peine d'être poursuivie dans les conditions de travail misérables qui étaient les siennes. Il décida d'écrire une missive enflammée, dans laquelle il expliquait vouloir fermer son cabinet. Sa protestation a suscité des réactions: plusieurs confrères et consœurs lui ont manifesté leur solidarité et les médias s'en sont emparés, d'abord dans le journal local (Sächsische Zeitung [2]), puis dans un hebdomadaire de portée nationale (Die Zeit [1]).

\section{A l'origine du problème: les traitements d'après les principes d'une économie planifiée}

Les problèmes de prise en charge médicale évoqués dans la presse découlent de la budgétisation des soins ambulatoires instaurée en Allemagne. En effet, le Dr Krause peut uniquement prescrire à ses patients (près de 1100 dont les résidents de plusieurs maisons de retraite) les prestations de santé que le budget imposé lui permet. Ces «objectifs économiques prévus» [2] répondent à des estimations moyennes, mais sont souvent épuisés avant le terme de la période de planifi- cation, précisément lorsque les maladies des patients ne se déroulent justement pas comme souhaité par les répondants des coûts. Le Dr Krause doit financer de sa poche les dépassements de budget.

Voici ses mots pour décrire l'impact de ce système sur son travail quotidien: "Nous perdons notre temps pour de la bureaucratie et sommes en conflit permanent avec notre association de médecins conventionnés» [2]. Tous les médecins conventionnés sont obligés d'être affiliés à une telle association (Kassenärztliche Vereinigung), placée sous surveillance ministérielle et chargée de répartir les sommes globales versées par les caissesmaladie. Elle doit donc répartir entre les différents médecins l'ensemble du budget qui, pour l'essentiel, provient de la contribution à l'assurance obligatoire à hauteur de $14,6 \%$ du salaire brut, et vérifier les besoins de chacun.

\section{Ecrasement administratif pour la justi- fication des décisions médicales}

Pour le Dr Krause, cette vérification s'est soldée en 2017 par trois contrôles des caisses en plus de ses journées de dix heures. Il a ainsi dû expliquer par écrit "pourquoi les bas de contention, la physiothérapie et les médicaments prescrits au patient X étaient nécessaires» [2] S'il ne peut pas justifier suffisamment la nécessité des traitements prodigués ou des médicaments prescrits, il doit procéder à leur remboursement: "Je paie maintenant, et de ma propre poche, des traitements que j'ai prodigués il y a près de trois ans et demi» [2], rapporte le Dr Krause au journaliste. L'avis des caisses-maladie et de son association de médecins conventionnés prime sur le fait de savoir si lui, en tant que médecin traitant, estime que les médicaments et les traitements étaient pertinents. Même sa mère (dont il a repris le cabinet 
en 2014) est confrontée pendant sa retraite à des actions récursoires des caisses avec des montants à cinq chiffres. La perte de motivation des médecins est facilement compréhensible lorsque leurs décisions thérapeutiques sont perpétuellement remises en question, à la lumière d'objectifs économiques planifiés. Le Dr Krause considère d'ailleurs que la liberté thérapeutique est inexistante et ajoute: "Je ne prescris aucun médicament par plaisir» [2]. Il s'inquiète, "car, à ce rythme, la prise en charge par les médecins de campagne risque de disparaitre prochainement" [2] - ce qui semble tout à fait plausible lorsqu'un médecin bien enraciné dans son village natal comme le Dr Krause a envie de tout arrêter.

\section{Budget limité $=$ actions récursoires et travail non rémunéré}

Les problèmes engendrés par la budgétisation des soins ne touchent pas seulement les médecins de campagne et les médecins de premier recours. Selon le Sächsische Zeitung, qui cite le porte-parole de l'association des médecins conventionnés de Saxe en lien avec le cas "d'un orthopédiste qui travaille gracieusement pendant certaines périodes suite à des dépassements de budget» [1], les caisses-maladie "ne mettraient à disposition qu'un budget déterminé pour la majeure partie des prestations médicales. Mais cela ne suffirait pas à indemniser entièrement toutes les prestations des médecins installés» [1].

Les déclarations du Dr Andreas Gassen, président de l'association fédérale des médecins conventionnés, luimême orthopédiste, vont dans le même sens. Dans un interview avec la revue Spiegel, il explique qu'«au quotidien, les budgets fixes ont effectivement pour conséquence d'obliger un médecin à calculer précisément ce qu'il prescrit et à quel patient, et combien de patients il peut se permettre» [3]. Ce qu'il évoque ensuite montre l'impact d'un budget global et la perte de ce qui devrait être une évidence: «Nous voulons juste que toute consultation avec un patient soit honorée, et surtout que le forfait de base soit versé pour chaque première consultation de chaque patient» [3]. Contraint, lui aussi, de devoir traiter "quasi gracieusement» [3] un patient sur huit qui vient dans son cabinet, il comprend tout à fait «que des collègues dans une situation similaire réfléchissent trois fois avant d'accepter les derniers patients qui frappent à cinq heures moins cinq à la porte du cabinet si leur bud- get est déjà épuisé» [3]. La budgétisation ne touche pas uniquement les patients en phase aiguë, mais se reflète également dans les temps d'attente pour obtenir un rendez-vous, jusqu'à 71 jours à Hambourg pour aller chez un cardiologue, contre seulement 19 pour les patients assurés en privé [4].

\section{La budgétisation: le début de la médecine à deux vitesses}

La budgétisation des soins ambulatoires impacte exclusivement les assurés des caisses-maladie publiques, soit près de $90 \%$ de la population allemande. Celui qui paie de sa poche ou qui est affilié à une assurance privée peut être soigné sans subir les restrictions budgétaires. Les effets de la budgétisation se concrétisent donc indirectement par une médecine à deux vitesses. L'orthopédiste A. Gassen rapporte par exemple que des massages médicalement pertinents, notamment contre la spasticité ou les troubles musculaires douloureux, peuvent être prescrits sans problème aux assurés privés. "Avec une assurance publique, un médecin doit impérativement vérifier à chaque prescription s'il dépasse son budget - en d'autres termes, s'il peut continuer de prescrire des prestations pour le traitement de tous ses autres patients sans risquer un recours (une procédure d'économicité)» [3].

A la question du Spiegel, qui aimerait savoir comment, selon le Dr Gassen, on pourrait rendre le système de santé plus juste, la réponse ne se fait pas attendre: "Très simplement: il suffirait de supprimer les budgets dans l'assurance-maladie obligatoire» [3]. Les expériences vécues par un médecin de campagne, le Dr Krause, et un orthopédiste, par ailleurs président de l'association fédérale des médecins conventionnés, le Dr Gassen, permettent donc de conclure que pour avoir une prise en charge médicale meilleure et plus juste en Allemagne, il suffirait de supprimer un principe dont l'introduction nous est présentée actuellement en Suisse comme un frein aux dépenses sans effets secondaires néfastes.

\section{Références}

1 Reinhard D. Ihm reicht es. Die Zeit. 17.11.2017.

2 Heinke M. Landarzt will seine Zulassung verbrennen. Sächsische Zeitung. 28.10.2017.

3 Interview avec Andreas Gassen dans la revue Spiegel 7/2018, p. 65-66; titre: «Das ist eine Tortur».

4 Spiegel online. 17.7.2011. Ärzte diskriminieren Kassenpatienten systematisch. 